\title{
Access to disease modifying treatments for rheumatoid arthritis patients
}

Rheumatoid arthritis (RA) is a severe disease that leads to joint destruction, loss of quality of life, work disability and premature mortality, as well as significant costs to the patient and to the health care system. Although effective therapeutic regimens are currently available, many patients fail to respond to these treatments, fail to sustain an initial response, or suffer from significant toxicity necessitating withdrawal of treatment. Moreover, remission is rare and curative treatment unknown. It is clear, therefore, that new treatments are needed.

Patients and doctors wish the best possible treatment to be prescribed for RA. The highest responsibility of doctors is that towards their patients. However, in an environment of increasing costs for medical care, doctors must also be cognisant of issues related to the costs of newly available treatments. In this context, it is helpful to establish a consensus of worldwide experts for the rational and successful use of such new therapeutic agents, even though such a consensus is provisional and will change as new data become available.

The newly developed disease modifying anti-rheumatic treatments seem to be a significant advance in the therapeutic armamentarium for RA. The presently available evidence shows that these agents lead to a significant reduction in the signs and symptoms of RA, a decrease in acute phase proteins and an increase in health related quality of life. As with all newly developed compounds, the long term efficacy and long term side effects of these new agents are as yet unknown. A method for a systematic collection and analysis of long term data on all such agents and controls is strongly recommended.

The use of these new agents should be under the supervision of physicians who are experienced in the diagnosis and treatment of RA, including the use of immunomodulatory agents. In addition, they should be experienced in the clinical assessment of RA treatment, including efficacy and long term observation for potential toxicities related to these drugs.

\section{Provisional consensus statement on TNF blocking agents in rheumatoid arthritis (March 1999)}

\section{Background}

A consensus group to consider the use of TNF (tumour necrosis factor) blocking agents was formed by an organising committee constituted of rheumatologists from the Universities of Erlangen, Leiden, and Vienna in cooperation with a number of universities in the United States. Pharmaceutical support was obtained from a number of companies, but these institutions had no part in the decisions regarding the specific programme nor with regard to the participants or attendees at this conference.

The approximately 80 rheumatologists and bioscientists from 22 countries who attended the consensus conference were chosen from a worldwide group of people felt to have experience or interest in the use TNF blocking treatment for RA. Unfortunately, the number of attendees and participants were limited so that not everyone who might have been appropriate could be invited.
While a great deal of knowledge regarding the use of TNF blocking agents in RA is as yet unknown (long term effectiveness and toxicity, effect on structural damage, use in early RA, etc), it was felt that a provisional statement regarding their use in RA would be very useful.

After initial discussion in small groups, two large group discussions and repeated drafts allowed repeated input from all participants and we feel that this document represents a good provisional consensus for the use of TNF blockade in RA. We, further, feel that it should be changed as additional evidence is gathered, presented in scientific forums and debated.

\section{Consensus}

INDICATION

Patients who are candidates for TNF blocking agents should have active rheumatoid disease despite a full and adequate trial of one or more DMARDs. Individual patients differ in the aggressiveness of their disease and its concomitant structural damage, the effect of their disease on their quality of life and the symptoms and signs engendered by their disease. All of these factors must be examined when considering TNF treatment for that patient, as must the toxicity of previous DMARD use. TNF blocking agents can be added to pre-existing methotrexate treatment.

The use of TNF blocking agents should be under the supervision of physicians who are experienced in the diagnosis and treatment of RA, including the use of immunomodulatory agents. In addition, they should be experienced in the clinical assessment of RA treatment including efficacy and long term observation of RA for potential toxicities related to immunomodulatory drugs.

REASONS FOR DISCONTINUATION

TNF blocking agents, when given in adequate doses and sufficiently frequent dosing regimens, should lead to significant, documentable improvement in symptoms, signs and/or laboratory parameters within 8 to 12 weeks. If such improvement has not occurred within this time frame, alternative treatments or regimens should be considered.

\section{WARNINGS}

TNF blocking agents should not be started or should be discontinued when serious infections occur, such as septic arthritis, infected prosthesis, pyelonephritis, postoperative abscesses, osteomyelitis, sepsis, systemic fungal infections, listeria or mycobacterial infections, etc. Treatment should only be resumed if such infections have been completely healed and the risk of recurrence is low.

\section{PRECAUTIONARY STATEMENTS}

The effect of TNF blockade is unknown in the following situations:

1 Lymphoma, lymphoproliferative disease and, possibly, other malignancies.

2 Chronic viral infections such as HIV, hepatitis B or C.

3 During pregnancy or lactation. 


\section{ADDITIONAL COMMENTS}

1 Rare cases of lupus-like disease have occurred in patients receiving TNF blocking agents and treatment should be stopped if there is clinical evidence of a lupus-like syndrome. There is thus far no evidence that RA patients who have positive ANA and/or ACL are susceptible to develop symptoms of drug induced lupus and treatment in this group of patients can probably be continued.

2 As TNF blocking agents are proved efficacious and their toxicity profile is demonstrated in patients with polyarticular juvenile chronic arthritis, these treatments should be used in that population.

3 The safety of primary vaccinations and live-attenuated vaccines during TNF blockade treatment is not known.

4 The safety of sequential use of different TNF blocking agents is unknown, but cross reactivity is at least theoretically possible.

\section{Summary}

TNF inhibitors seem to be promising therapeutic agents for RA. The use of these new agents will require physicians experienced in the diagnosis, treatment and assessment of RA. These physicians will need to make long term observations for efficacy and safety. Further considerations when using TNF blocking agents in this disease must include cost issues and recognition that data in subpopulations are still being acquired. It is hoped that this statement, which is based upon the best evidence available at the time of its creation and modified by expert opinion, will facilitate the optimal use of these agents for our patients with RA.

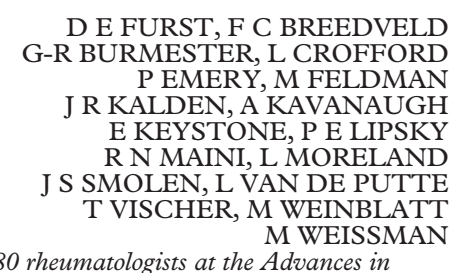

representing the approximately 80 rheumatologists at the Advances in Targeted Therapies TNF Blockade in Clinical Practice.

Professor Michael Doherty acted as guest editor for this article.
Supported by unrestricted educational grants from: Amgen, Centocor, Inc, Immunex Corp, Knoll AG, Schering Plough, Wyeth Ayerst.

None of the 17 primary authors are under retainer as consultants to sponsors interested in TNF $\alpha$ blocking treatment, although they may have received grants for research (basic and/or clinical) from the sponsor.

\section{Selected list of references}

Elliott MJ, Maini RN, Feldmann M, et al. Treatment of rheumatoid arthritis with chimeric monoclonal antibodies to tumor necrosis factor a. Arthritis Rheum 1993;36:1681-90.

Elliott MJ, Maini RN, Feldmann M, et al. Randomised double-blind comparison of chimeric monoclonal antibody to tumor necrosis factor a antibody (cA2) versus placebo in rheumatoid arthritis. Lancet 1994;344:1105-10.

Maini RN, Elliott MJ, Charles PJ, et al. Immunological intervention reveals reciprocal roles for TNFa and IL-10 in rheumatoid arthritis and SLE. Springer Semin Immunopathol 1994;16:327-36.

Maini RN, Elliott MJ, Brennan FM, et al. Monoclonal anti-TNFa antibody as a probe of pathogenesis and therapy of rheumatoid disease. Immunol Rev 1995;144:195-223.

Feldmann M, Elliott MJ, Woody JN, et al. Anti-tumor necrosis factor-alpha therapy of rheumatoid arthritis. Adv Immunol 1997;64:283-350.

Moreland LW, Baumgartner SW, Schiff MH, et al. Treatment of rheumatoid arthritis with a recombinant human tumor necrosis factor receptor (p75)-Fc fusion protein. N Engl J Med 1997;337:141-7.

Kalden-Nemeth D, Grebmeier J, Antoni C, et al. NMR monitoring of rheumatoid arthritis patients receiving anti-TNFa monoclonal antibody therapy. Rheumatol Int 1997;16:249-55.

Maini RN, Breedveld FC, Kalden JR, et al. Therapeutic efficacy of multiple intravenous infusions of anti-tumor necrosis factor a monoclonal antibody combined with low-dose weekly methotrexate in rheumatoid arthritis. combined with low-dose weekly met

Paleolog EM, Young S, Stark AC, et al. Modulation of angiogenic cascular endothelial growth factor by tumor necrosis factor a and interleukin-1 in rheumatoid arthritis. Arthritis Rheum 1998;41:1258-65.

Moreland L. Soluble tumor necrosis factor receptor ( $\mathrm{p} 75$ ) fusion protein (ENBREL) as a therapy for rheumatoid arthritis. Rheum Dis Clin North Am 1998;24:579-91.

Fenner $\mathrm{H}$. Immunopharmacologic profile and therapeutic prospects of anti-TNF-alpha therapy. Z Rheumatol 1998;57:294-7.

Felson D, Lafyatis R, Korn J. Rheumatology. Biologic agents-is the promise realised? Lancet 1998;352 (suppl 4):s25.

Kavanaugh AF. Anti-tumor necrosis factor-alpha monoclonal antibody therapy for rheumatoid arthritis. Rheum Dis Clin North Am 1998;24:593-614.

Anonymous. New drugs for rheumatoid arthritis. Med Lett Drugs Ther 1998; 40:110-12.

Goldenberg MM. Entanercept, a novel drug for the treatment of patients with severe, active rheumatoid arthritis. Clin Ther 1999;21:75-87.

Weinblatt E, Kremer JM, Bankhurst AD, et al. A trial of etanercept, a recombinant tumor necrosis factor receptor: $\mathrm{Fc}$ fusion protein, in patients with rheumatoid arthritis receiving methotrexate. N Engl J Med 1999;340: 253-9. 